CDPfer

SciencePark Research, Organization \& Counseling

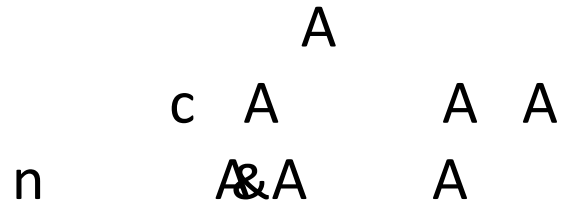

W AA3127[ A $^{1} 9$ 714A

\section{A A A A fb A A A A $\quad$ A $3127[\mathrm{~A}$}

\title{
Virtual reality (VR) technologies in education of industrial design
}

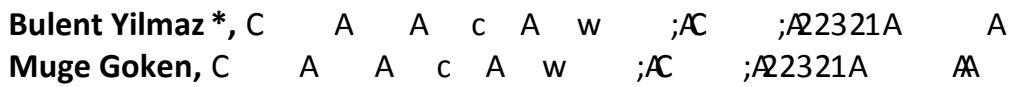

Suggested Citation:

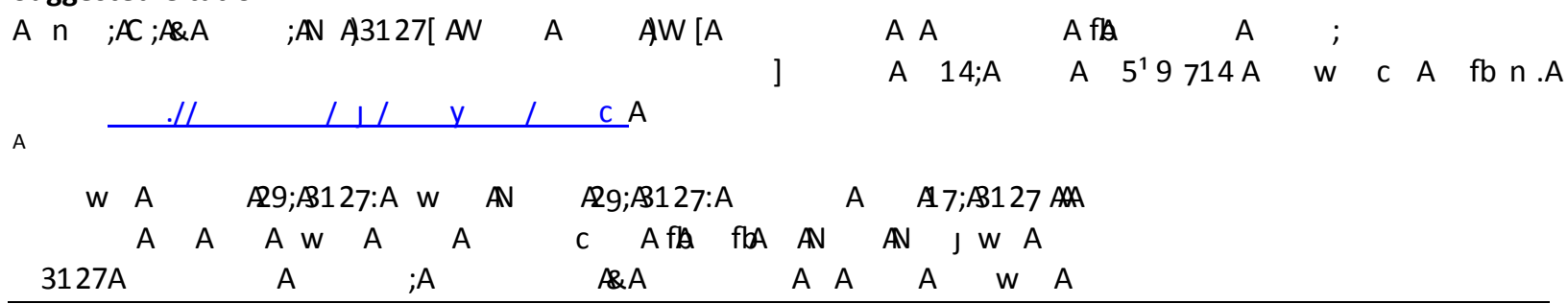

Abstract

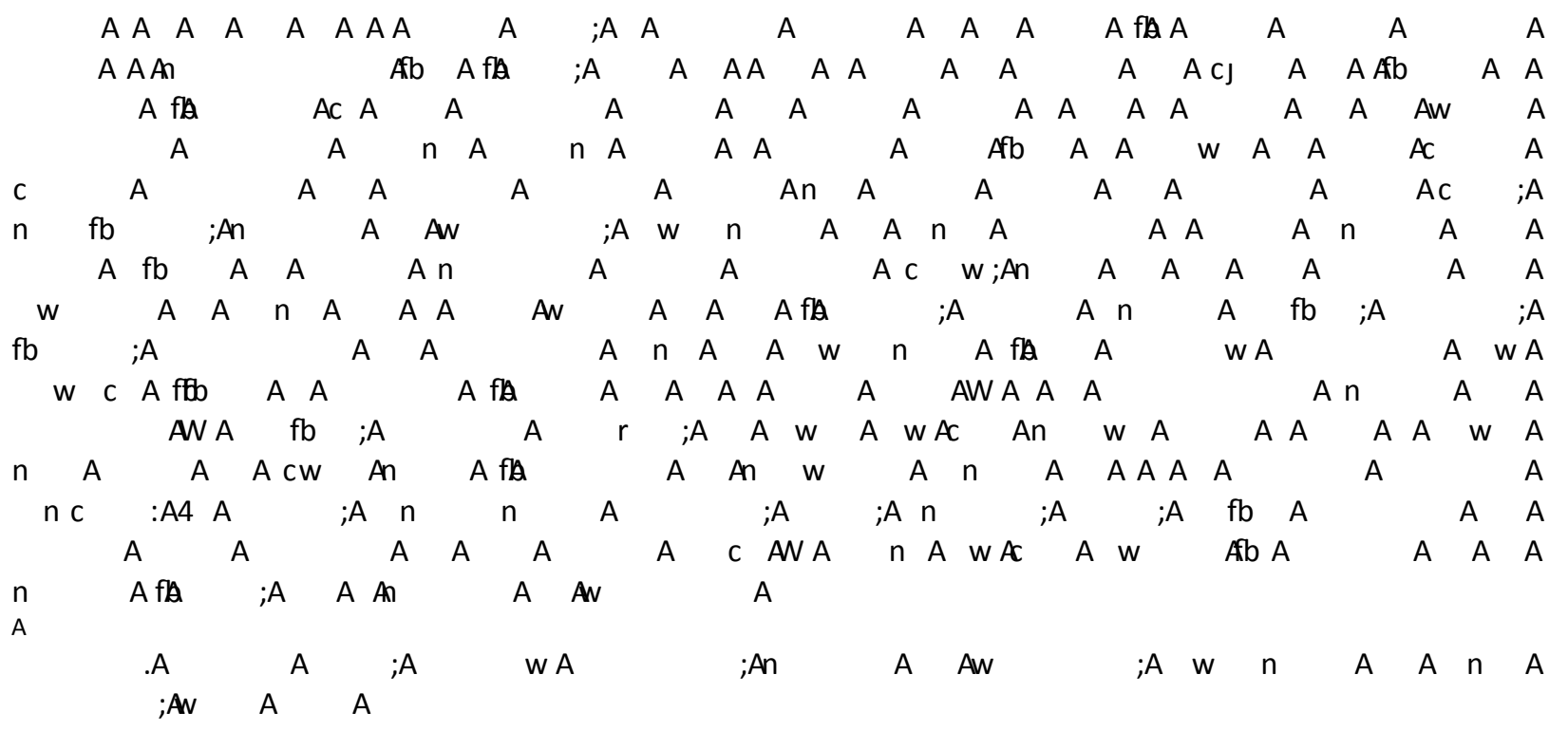

\footnotetext{
$\begin{array}{llllllllllllll}\text { ABBulent Yilmaz, G } & \text { A } & \text { A } & \text { A } & \text { AG } & ; A & \mathrm{n} & \text { A flA } & \text { A } & ; A C & \text { A } & \text { A } & \text { c } & \text { A }\end{array}$

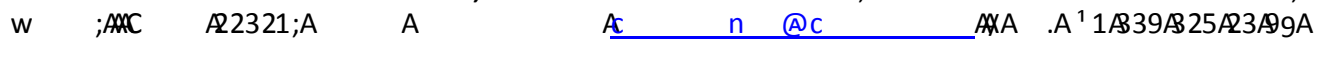




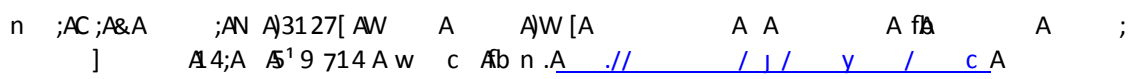

\section{IntroductionA}

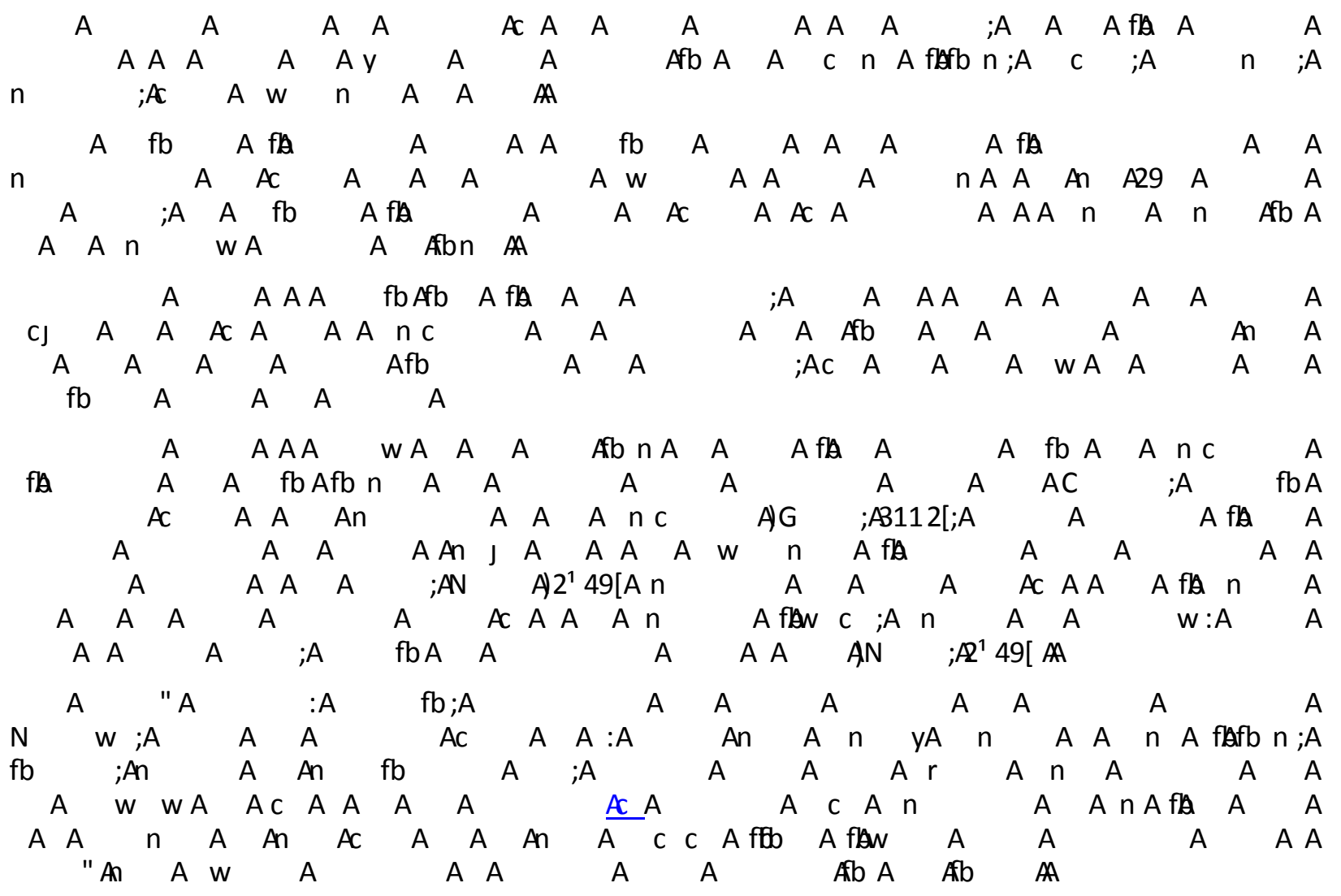

\section{Virtual Reality}

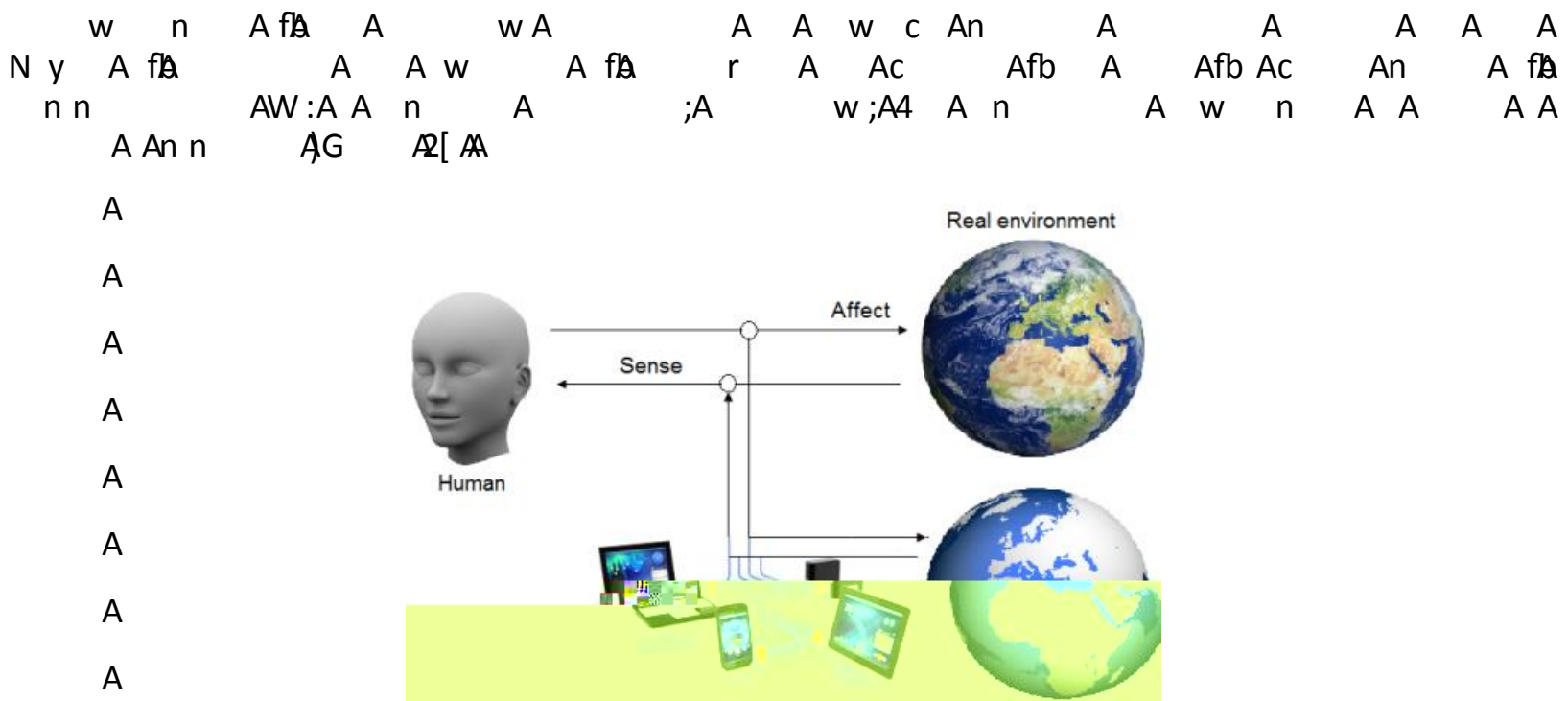

A

G AA A A A A 


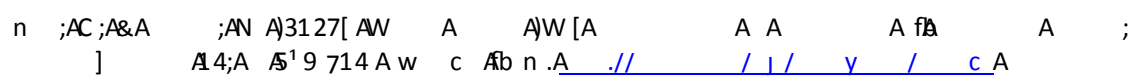

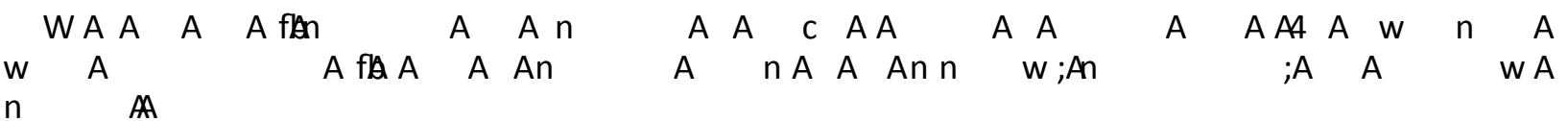

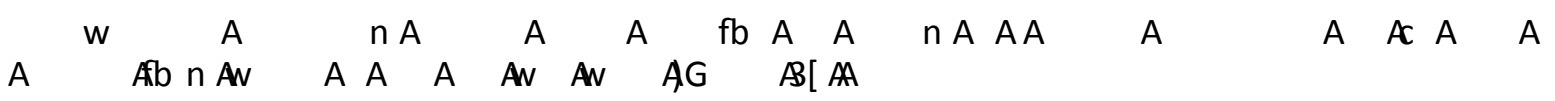
A

A

A

A

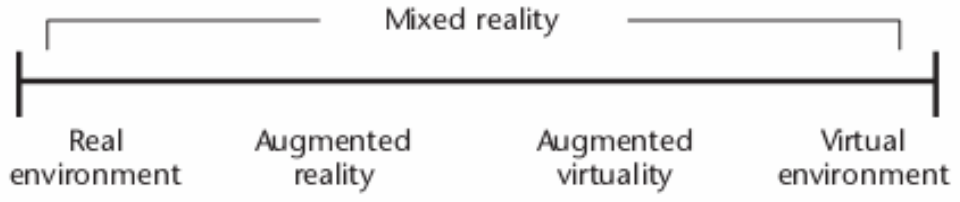

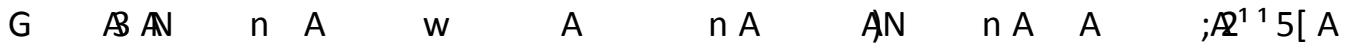

A

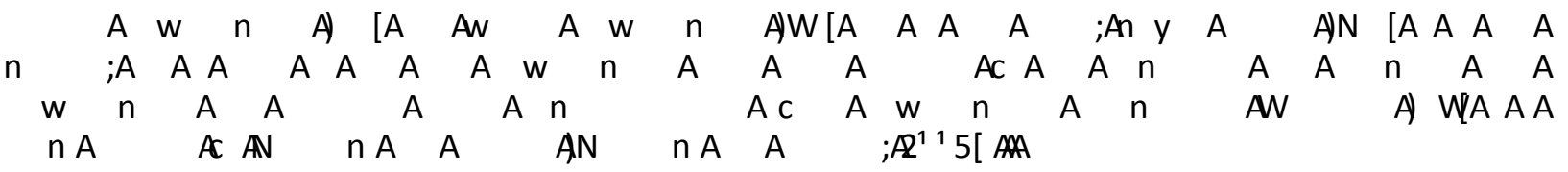

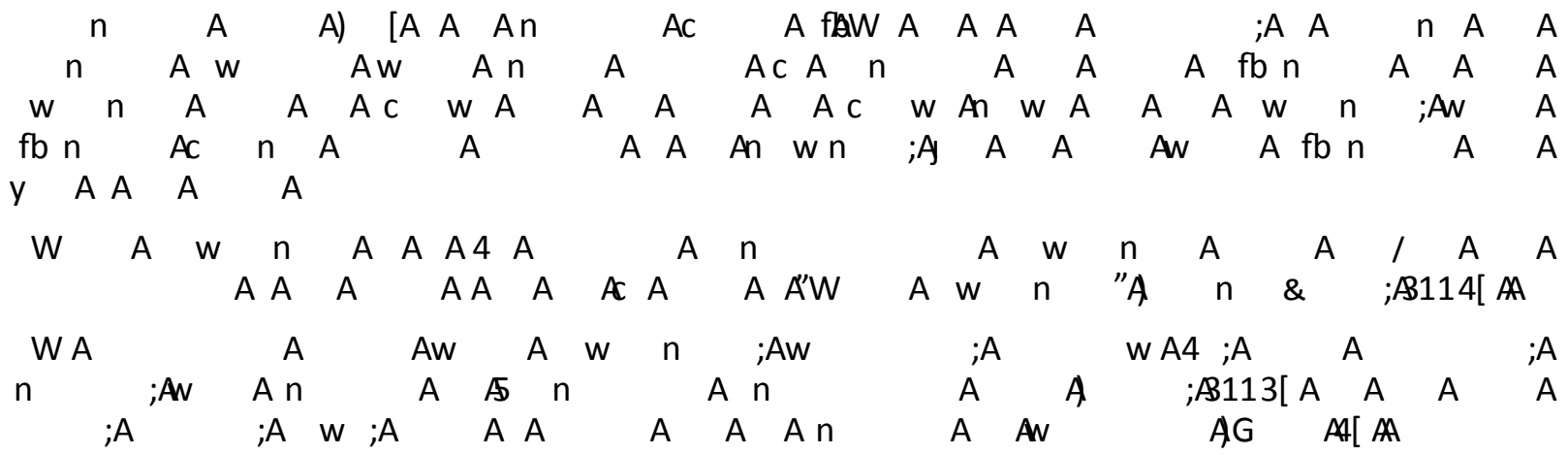

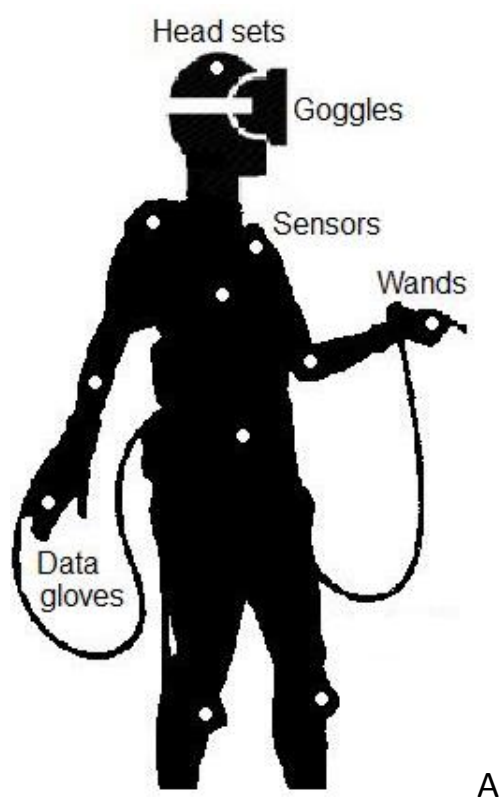

$G \quad A A \quad A \quad A B b$ AN A

500 


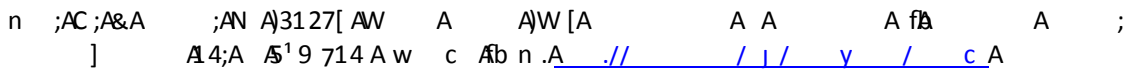

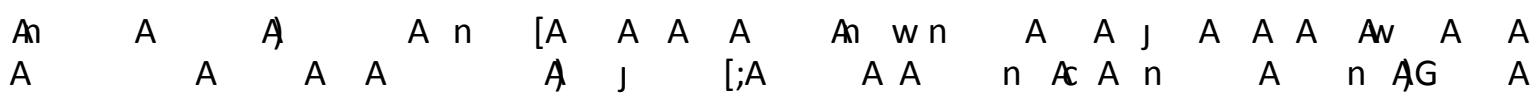
$5[A$
A

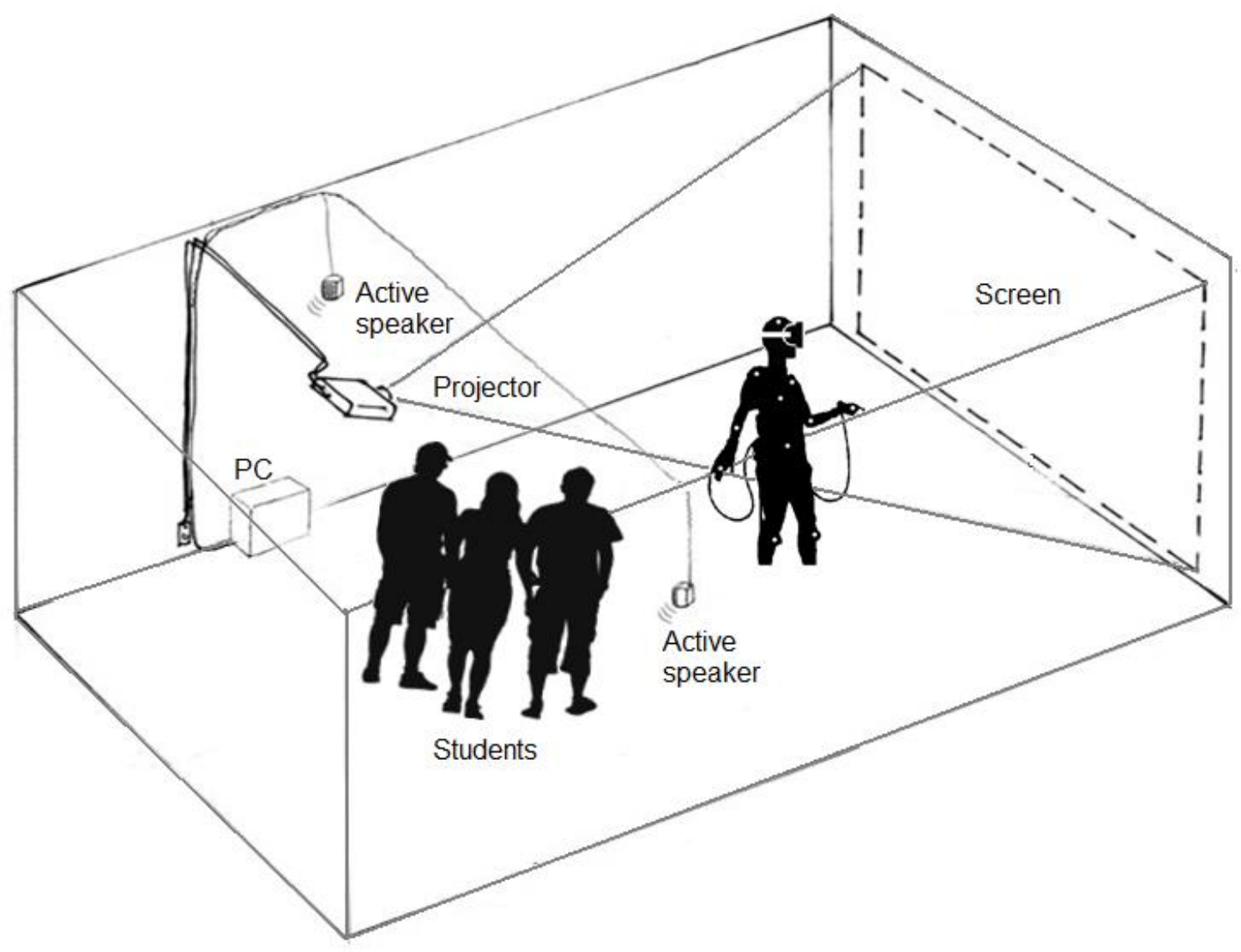

A

G $\quad A A C$ Ar $n \quad A$ FAN A

AWA AfB A $n$ A flall A A An A A A $\cap$ A A Afb A

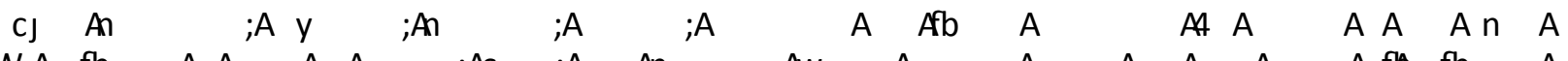

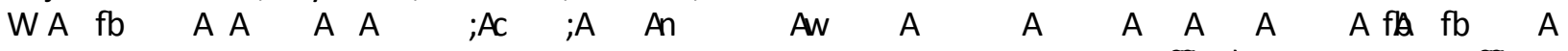

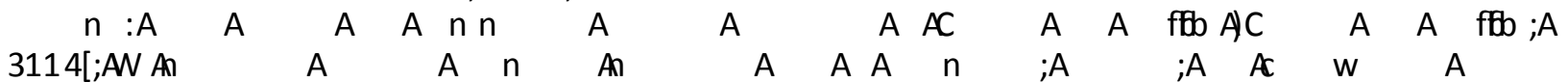

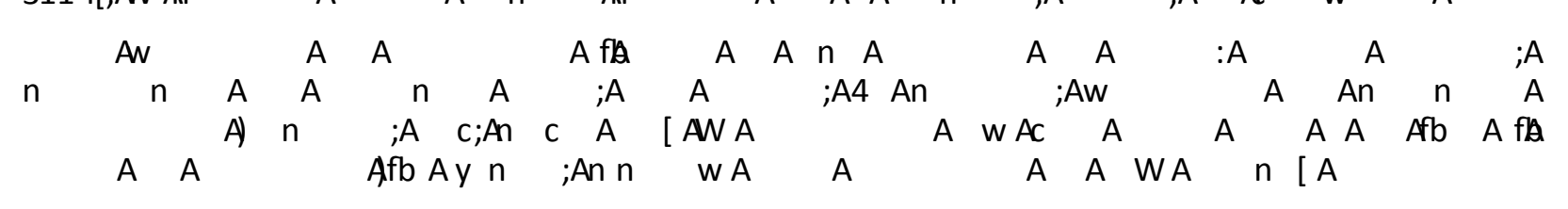

\section{Virtual Reality in Education}

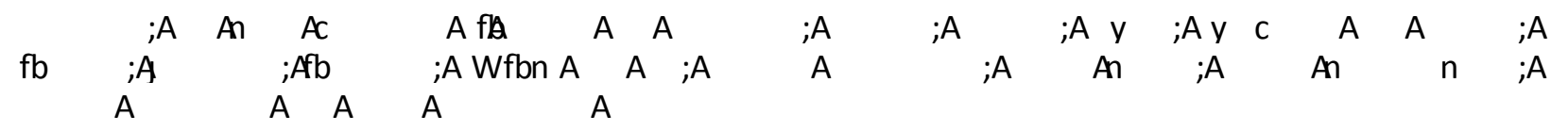




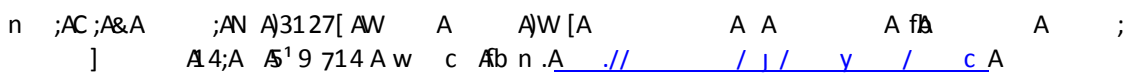

A A $A A A$ fb A AA W A $\cap A$ A A

;An A A

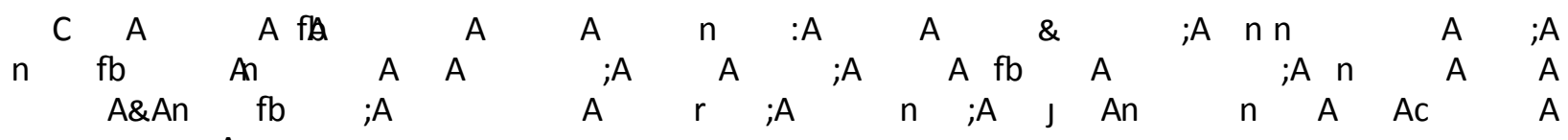
$n \quad n \quad A$

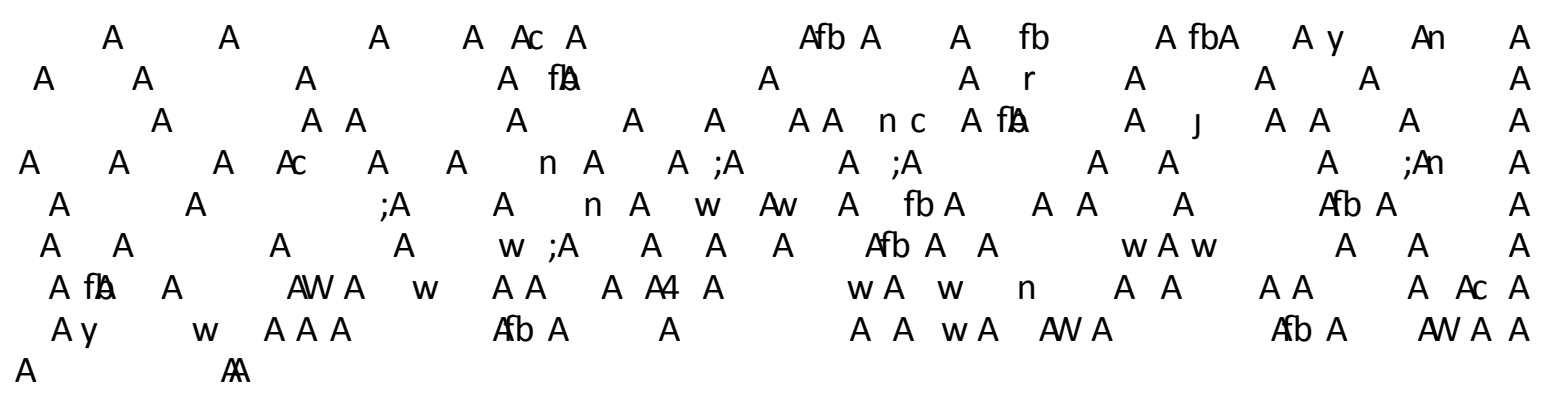

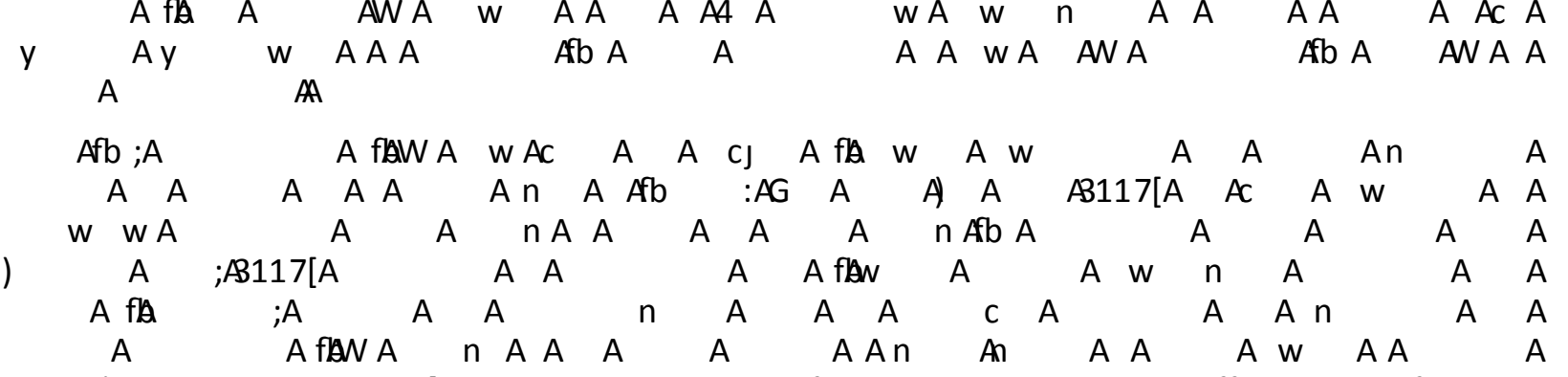

A) $A$ A $\quad$ A3119[A $\quad A \quad A \quad A f b A$ A $A A$ A ftb A Afb $n$ A

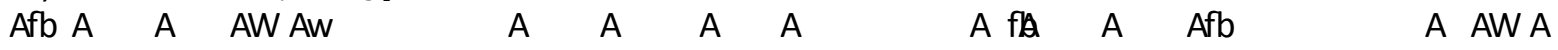

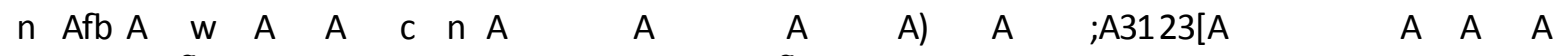

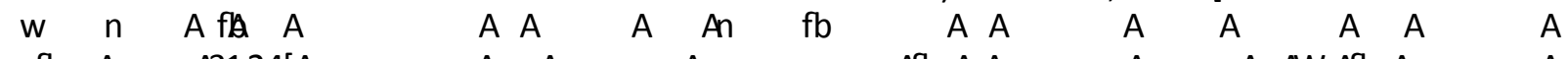

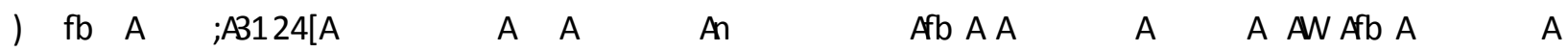
A $A$ A A $A$ A fla A A A A A A A Ac A fb A

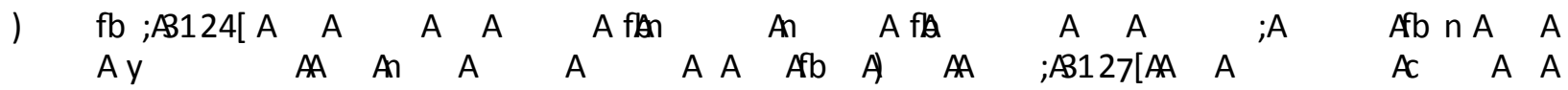

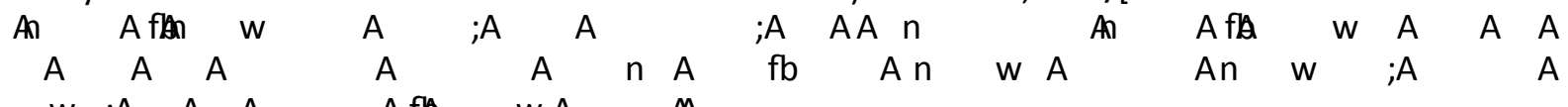

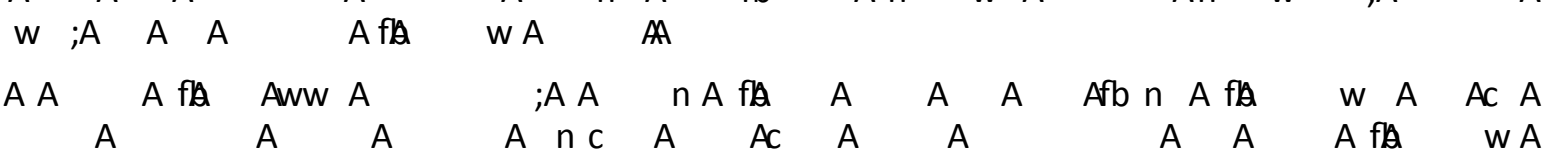
A

4. ConclusionsA

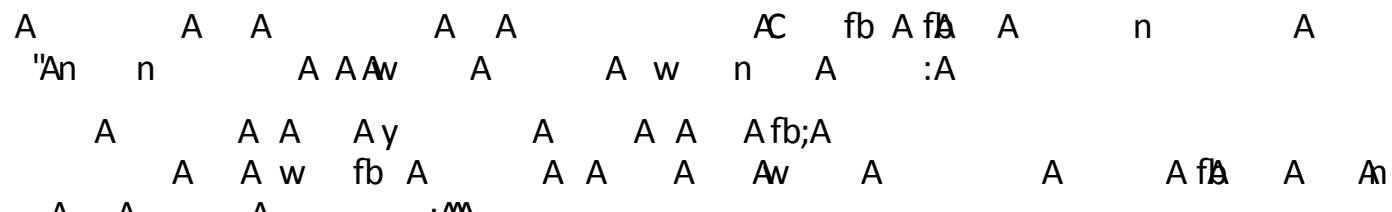

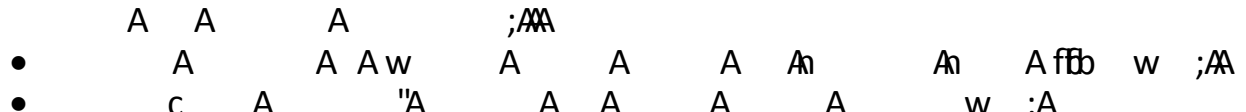

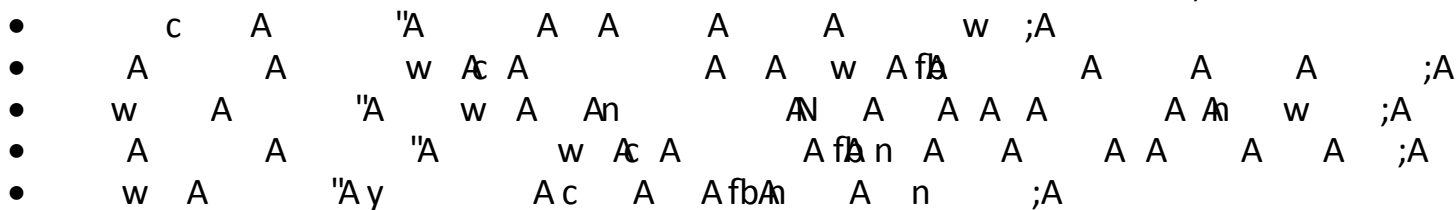

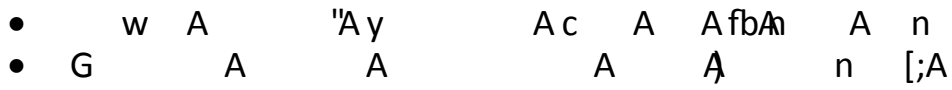

502 


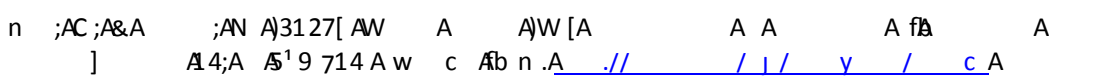

- G A An A A Ac A A A A A A J A A :A C;A

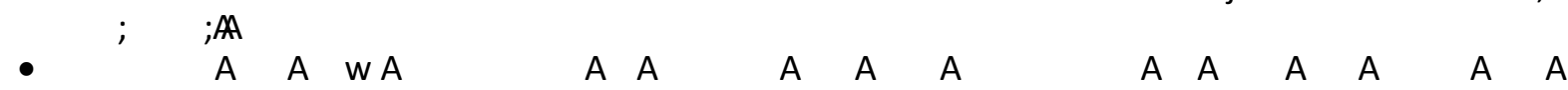

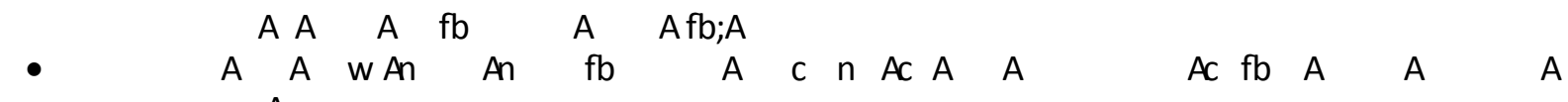
- A A wa An a A A A Afla n an afba

\section{ReferencesA}

C A ; $\quad$ \&A ftb $A ; A 3114\left[A^{\prime \prime}\right.$

G A ;A]3112[ $A^{\prime \prime} \quad$ A $A$

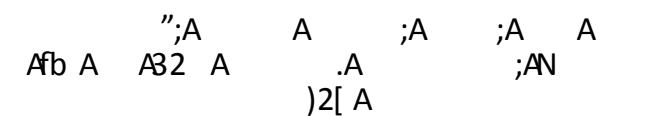

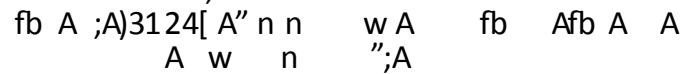
$\mathrm{fb} A ; A \quad f b$ AW;A w "W $\mathrm{A} ; \mathrm{A}) 3124\left[; \mathrm{A}^{\prime}\right.$

A A A

A AN A A A A An $\quad A f b$ AN 12322 A A

A $A$ A

$N \quad n A ; A \& A \quad A G ; A) 2^{11} 5\left[A \& A\right.$ y $n$ A flan y $A \begin{array}{llllllll}A & \text { AW } & A & \text {;A } & A & A & \text { fb } & A\end{array}$

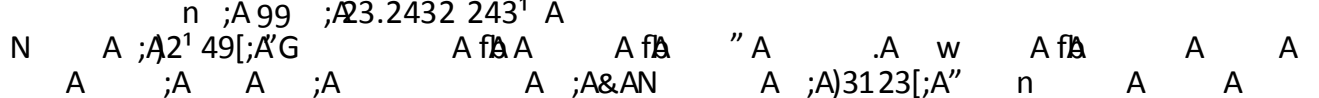

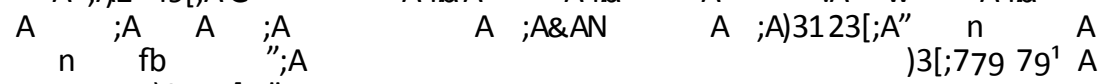
$\begin{array}{cccccc}A N ; A) 3115\left[A^{\prime \prime}\right. & A C^{\prime A} & A & A & A & A \\ w & \text { Afb A A Y }\end{array}$

n A ;A\&A A C;A]3114[A A fla $\cap$. AN A fb A C A

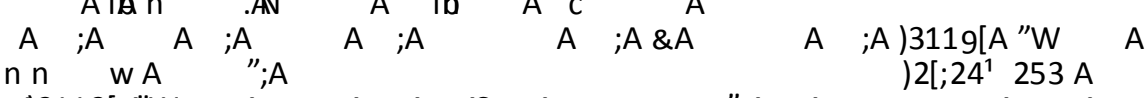

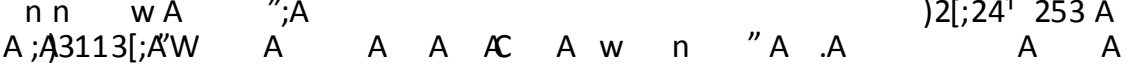

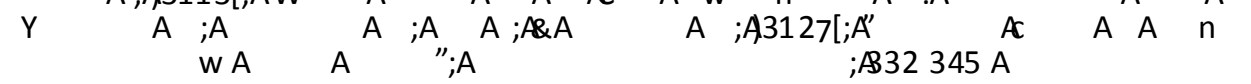

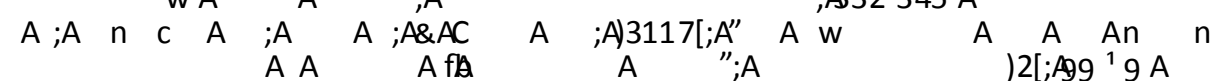

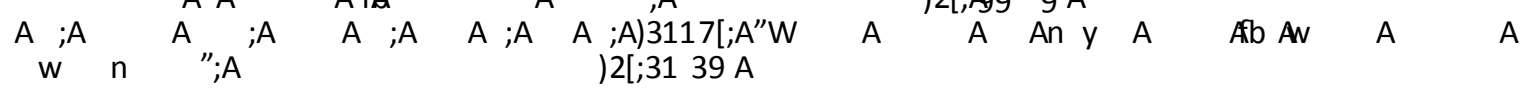

